\title{
Study on Applicability of Rare Earth High-temperature Superconducting Wires to Superconducting Magnet for Maglev System
}

\author{
Masafumi OGATA \\ Senior Researcher,
}

\author{
Katsutoshi MIZUNO \\ Researcher,
}

Cryogenic Systems, Maglev Systems Technology Division

\author{
Yuuki ARAI \\ Researcher,
}

Hitoshi HASEGAWA, Ph.D.

Senior Researcher,
Takashi SASAKAWA, Ph.D.

Senior Researcher,

Laboratory Head, Electromagnetic Applications, Maglev Systems Technology Division
Ken NAGASHIMA, Ph.D.

Senior Researcher,

Laboratory Head, Cryogenic Systems, Maglev Systems Technology Division

Rare earth high-temperature superconducting wire (RE wire) has electrical and mechanical properties superior to those of conventional superconducting wire. It was clarified that a superconducting magnet with a simpler structure and higher reliability than the conventional type could be achieved for maglev system by applying this type of wire. A characteristic test was then carried out for RE wire, and an electric properties test was run on a trial coil of the wire in a small racetrack shape. The electrical properties of $R E$ wire formations varying from a single wire shape to a wound coil shape were thus confirmed. The results obtained are expected to be useful to allowing the application of $R E$ wire to superconducting magnet for maglev system.

Keywords: RE, HTS, wire, SCM, superconducting magnet, maglev

\section{Introduction}

Rare earth high-temperature superconducting wire ( $\mathrm{RE}$ wire) has electrical and mechanical properties superior to those of conventional wire. It was clarified that a superconducting magnet with a simpler structure and higher reliability than the conventional type could be achieved for maglev system by applying this type of wire. A characteristic test was then carried out for RE wire, and an electric properties test was run on a trial coil of the wire in a small racetrack shape. The electrical properties of $\mathrm{RE}$ wire formations varying from a single wire shape to a wound coil shape were thus confirmed. The results obtained are expected to be useful to allowing the application of $\mathrm{RE}$ wire to superconducting magnet for maglev system.

\section{High-temperature superconducting wire}

Figure 1 shows a typical representation of the critical temperature $\left(T_{c}\right)$ for superconducting wire. As temperature decreases, the resistance of general metal materials gradually approaches zero. However, the resistance of superconducting wire disappears at a certain temperature with transmission from a normal conducting state to a superconducting state. This temperature is called the critical temperature $\left(T_{c}\right)$, and acts as a representative index for superconducting materials. As there are similar indices (e.g., the critical magnetic field $\left(B_{c}\right)$ and the critical current density $\left(J_{c}\right)$ (Fig. 2)), the superconducting state is limited to the area satisfying these three critical conditions. For studies on application, it is therefore important to verify each of the critical conditions for superconducting wire.

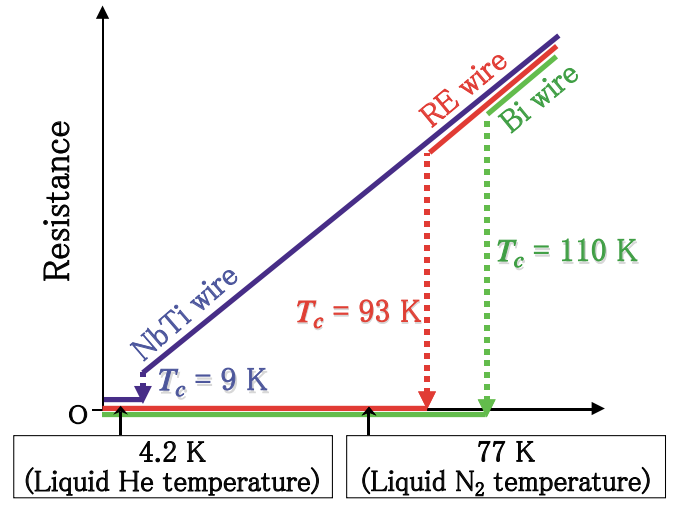

Temperature

Fig. 1 Image of critical temperature $T_{c}$ for superconducting wire

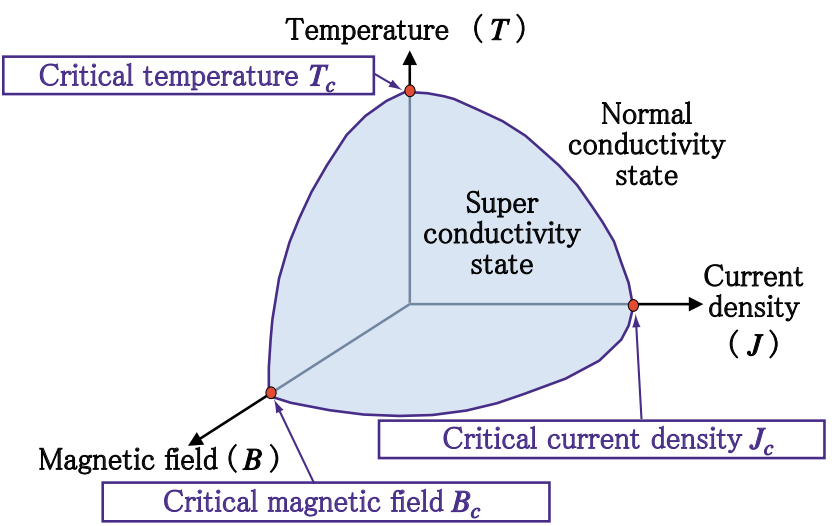

Fig. 2 Critical condition for superconductivity state 
High-temperature superconducting wire exhibits superconductivity at higher temperatures than conventional metal superconducting wire. In general, high-temperature superconducting wire has a $T_{c}$ value higher than the temperature of liquid nitrogen. There are two kinds of hightemperature superconducting wire - $\mathrm{Bi}$ wire and $\mathrm{RE}$ wire - in practical use today. Both Bi wire, which includes bismuth, and RE wire, which includes rare earth elements such as yttrium, are copper oxide high-temperature su-

Table 1 Specifications of HTS wire

\begin{tabular}{|l|c|c|}
\hline & Bi wire $^{(*)}$ & RE wire $^{(* *)}$ \\
\hline Width & $4.3 \mathrm{~mm}$ & $4.4 \mathrm{~mm}$ \\
\hline Thickness & $0.23 \mathrm{~mm}$ & $0.20 \mathrm{~mm}$ \\
\hline Critical current $(77 \mathrm{~K})$ & $<180 \mathrm{~A}$ & $<90 \mathrm{~A}$ \\
\hline Critical tensile stress & $130 \mathrm{MPa}$ & $200 \mathrm{MPa}$ \\
\hline Critical bend diameter & $70 \mathrm{~mm}$ & $25 \mathrm{~mm}$ \\
\hline
\end{tabular}

* Sumitomo Electric Industries: DI-BSCCO Type H

** American Superconductor: Type 344C

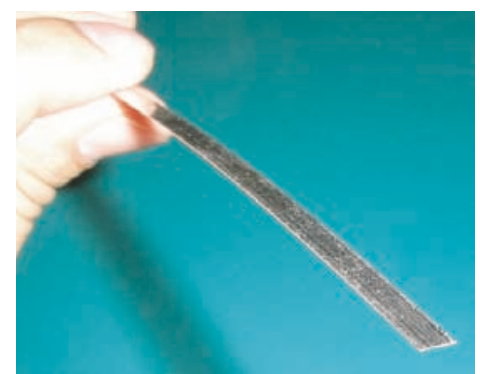

Fig. 3 RE high-temperature superconducting wire

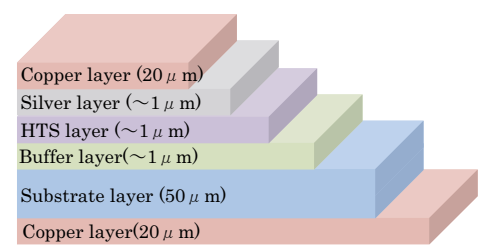

Fig. 4 Typical structure of RE wire perconducting wires. Since the $T_{c}$ value of Bi wire is $110 \mathrm{~K}$ and that of $\mathrm{RE}$ wire is $93 \mathrm{~K}$, both types of wire can be superconducting state by cooling with liquid nitrogen.

Table 1 shows the main specifications of $\mathrm{Bi}$ wire and $\mathrm{RE}$ wire, and indicates that the mechanical characteristics of $\mathrm{RE}$ wire are at least as good as those of Bi wire. As a result of recent global research and development, the characteristics of $\mathrm{RE}$ wire (such as piece wire length, critical current and manufacturing cost) have improved remarkably, giving it the potential status of second-generation high-temperature superconducting wire and successor to Bi wire. Although Bi wire currently takes precedence for applications such as motors and cables, it is conceivable that the application of $\mathrm{RE}$ wire will become the mainstream in the near future.

Figure 3 shows the appearance of RE wire, and Fig. 4 shows its typical structure. It has a tape-like appearance, and features a laminated film structure on a non-magnetic substrate to provide mechanical strength.

\section{Advantages of applying RE wire to superconducting magnet for maglev system}

Figure 5 outlines the advantages of applying RE wire to a conventional superconducting magnet using $\mathrm{NbTi}$ wire for a maglev system. Cryocooler-based conduction cooling is made practical by raising the operating temperature of the superconducting coils, thereby eliminating the need for liquid helium/liquid nitrogen coolants and the related tanks and piping. In addition, the conventional cryocooler can be replaced with a small lightweight type featuring low energy consumption. As a result of increased thermal capacity with high-temperature operation, the stability of superconducting coils against thermal disturbance is improved. In addition, if the operating temperature of a superconducting coil exceeds $50 \mathrm{~K}$, no radiation shield is necessary, and downsizing of the outer vessel's cross section can be realized [1].

It was thus clarified that the application of $\mathrm{RE}$ wire allows superconducting magnet design with a simpler structure and improved stability.

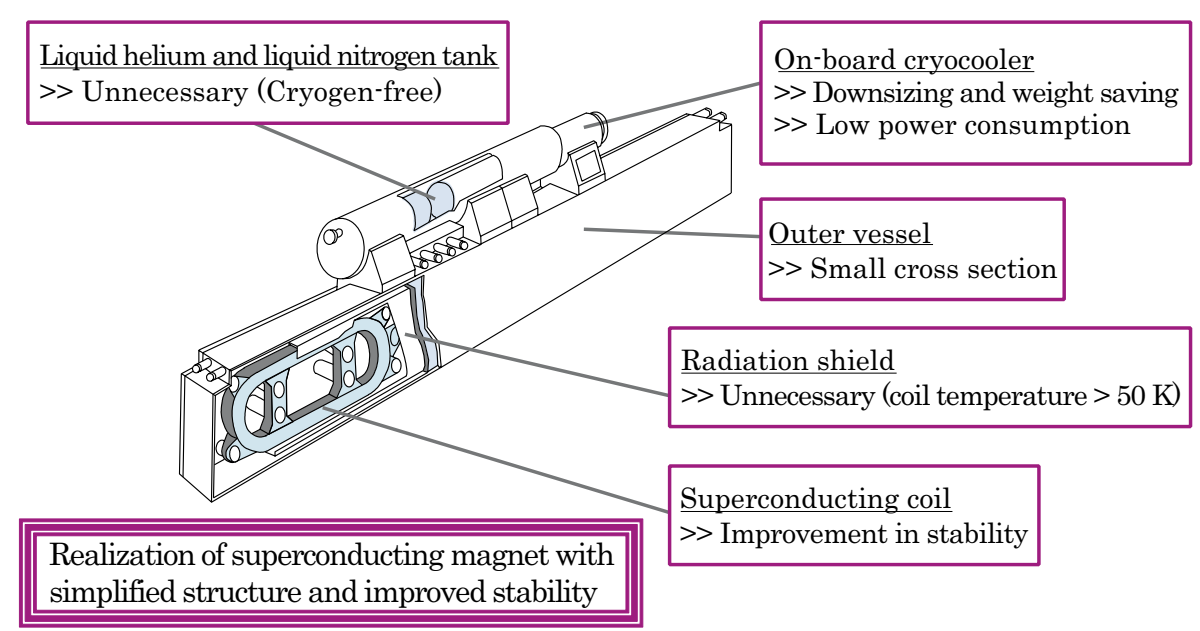

Fig. 5 Merits of applying RE wire to superconducting magnet for maglev system (Figure is schematic view of superconducting magnet with $\mathrm{Nb}-\mathrm{Ti}$ coils) 


\section{Results of transport current properties testing on RE wire}

To allow the application of $\mathrm{RE}$ wire to superconducting coils, it is essential to confirm its quantitative transport characteristics in relation to environmental variables such as temperature and magnetic fields. Due to a lack of published studies on these basic characteristics of $R E$ wire then, we developed a system to evaluate its transport current characteristics under the conditions of current $(I)$, magnetic field $(B)$, magnetic field angle $(\theta)$, temperature $(T)$ and bend diameter $(\phi)$. The system consists of an I-B- $\theta$-T test device (Fig. 6) and an I- $\phi$ test device (Fig. 7). The I-B- $\theta$-T test device works with a maximum current of $1000 \mathrm{~A}$, a maximum magnetic field of $5.5 \mathrm{~T}$ and a minimum temperature of $10 \mathrm{~K}$. Since the aspect ratio of the wire section is large and the crystal structure of the su-

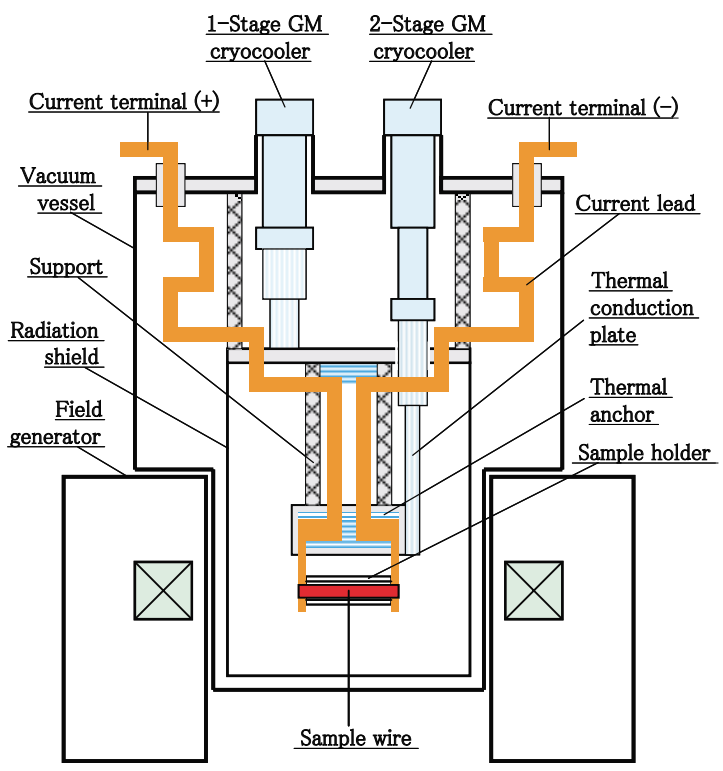

Fig. 6 Schematic of I-B- $\theta$-T test device

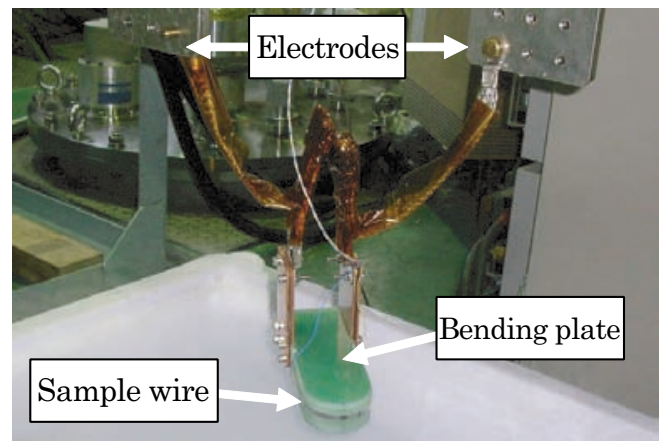

Fig. 7 I- $\phi$ test device

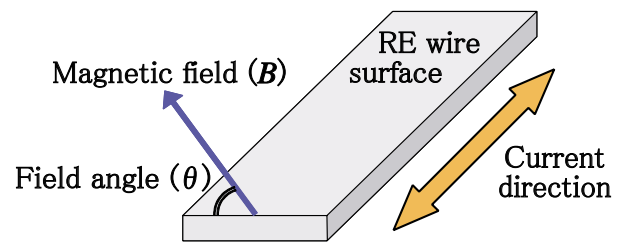

Fig. 8 Definition of $\theta$ perconducting layer is highly oriented, RE wire has high anisotropy in terms of transport current characteristics in magnetic fields. Accordingly, the device supports the evaluation of anisotropy for magnetic field angle $\theta$ formed by the wire surface and $B$ (Fig. 8). The I- $\phi$ test device can also evaluate the bend diameter dependence of $\mathrm{RE}$ wire's transport current characteristics in liquid nitrogen at 77 $\mathrm{K}[2]$.

Using the I-B- $\theta-\mathrm{T}$ test device, the characteristics of RE wire shown in Table 1 were evaluated. Figure 9 shows the magnetic field dependence of $I_{c}$ at a magnetic field angle of 0 degrees. This outcome indicates that $I_{c}$ simply decreases with higher temperatures and in higher mag-

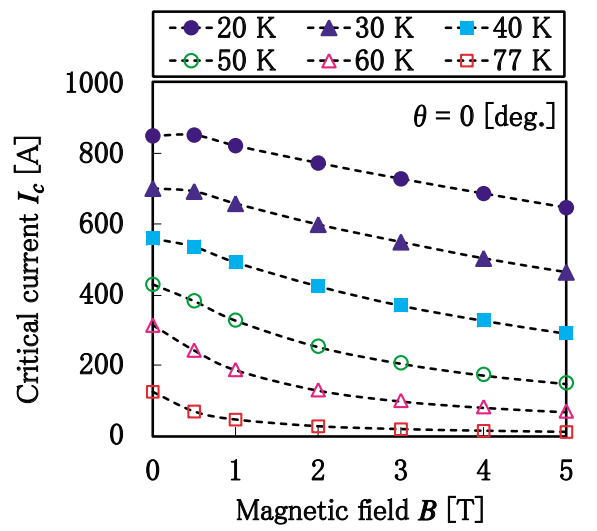

Fig. 9 Magnetic field dependence of critical current for RE wire

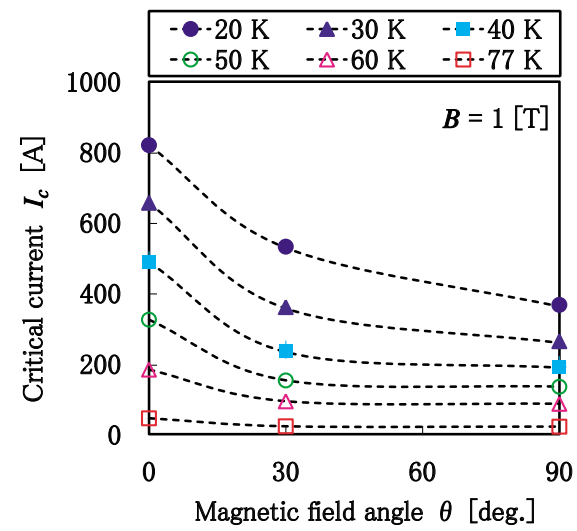

Fig. 10 Magnetic field angle dependence of critical current for RE wire

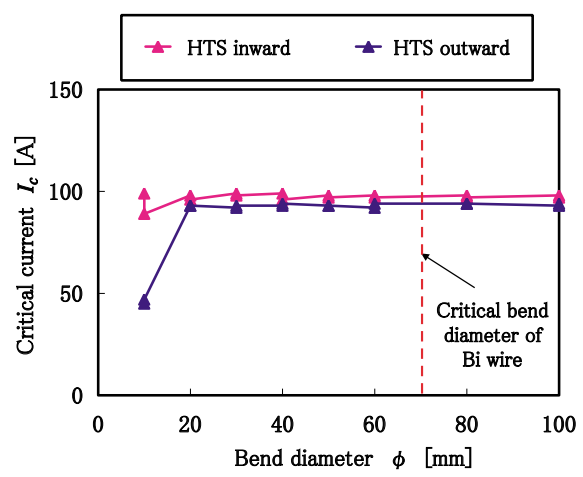

Fig. 11 Bend diameter dependence of critical current for RE wire 
netic fields. Figure 10 shows the magnetic field angle dependence of $I_{c}$ in a magnetic field of $1 \mathrm{~T}$. The results indicate that $I_{c}$ reaches its maximum value for a magnetic field angle of 0 degrees and its minimum value for a magnetic field angle of 90 degrees. This means that the magnetic field angle causes a difference of around $60 \%$ in $I_{c}$ even under the same temperature and magnetic field conditions. As this anisotropy of transport current characteristics is problematic for the application of $\mathrm{RE}$ wire to superconducting equipment, $\mathrm{RE}$ wire manufacturers are currently pursuing research and development such as doping an artificial pinning center to address the issue [3].

Figure 11 shows the results of testing the bend diameter dependence of $I_{c}$ for RE wire as outlined in Table 1 using the I- $\phi$ test device with a liquid nitrogen temperature of $77 \mathrm{~K}$. The outcome indicates that there is no deterioration of $I_{c}$ until a bend diameter of $20 \mathrm{~mm}$, which is much smaller than the critical bend diameter of $70 \mathrm{~mm}$ for Bi wire as shown in Table 1. This characteristic against the bending transformation of $\mathrm{RE}$ wire is very advantageous in terms of coil design flexibility and ease of wire handling. It is also apparent that the deterioration of $I_{c}$ with the outward high-temperature superconducting layer is remarkable compared to that of the inward layer.

\section{Results of transport current properties testing on an RE coil}

Using $100 \mathrm{~m}$ of $\mathrm{RE}$ wire with the characteristics outlined in Table 1, a small racetrack-shaped RE coil was produced with an inside diameter of $100 \mathrm{~mm}$ and a straight part measuring $150 \mathrm{~mm}$. Figure 12 shows the coil, which was formed from a single pancake coil, and Table 2 outlines its specifications.

We carried out an $I_{c}$ evaluation test on this RE coil using the I-B- $\theta$-T test device under self-magnetic field conditions. Figure 13 shows the transport current dependence of the magnetic fields for the RE coil at $30 \mathrm{~K}$. The results show the presence of magnetic fields of $0.85 \mathrm{~T}$ at the innermost layers at the center of the curved part and $0.3 \mathrm{~T}$ at the coil center. Figure 14 shows the temperature dependence of $I_{c}$ for the RE coil, and confirms that the value decreases with higher temperatures as seen with the $I_{c}$ behavior of RE wire itself. In the case of this coil, $I_{c}$ at $77 \mathrm{~K}$ decreased to approximately a one-fifth of the value seen at $30 \mathrm{~K}$.

Table 2 Specifications of racetrack shape RE coil

\begin{tabular}{|l|c|}
\hline Wire length & $100 \mathrm{~m}$ \\
\hline Wire width & $4.4 \mathrm{~mm}$ \\
\hline Wire thickness & $0.2 \mathrm{~mm}$ \\
\hline Inside diameter & $100 \mathrm{~mm}$ \\
\hline Outside diameter & $175 \mathrm{~mm}$ \\
\hline Straight part length & $150 \mathrm{~mm}$ \\
\hline Number of turns & 138 turns \\
\hline Number of pancakes & 1 single pancake \\
\hline Inductance & $6.8 \mathrm{mH}$ \\
\hline
\end{tabular}

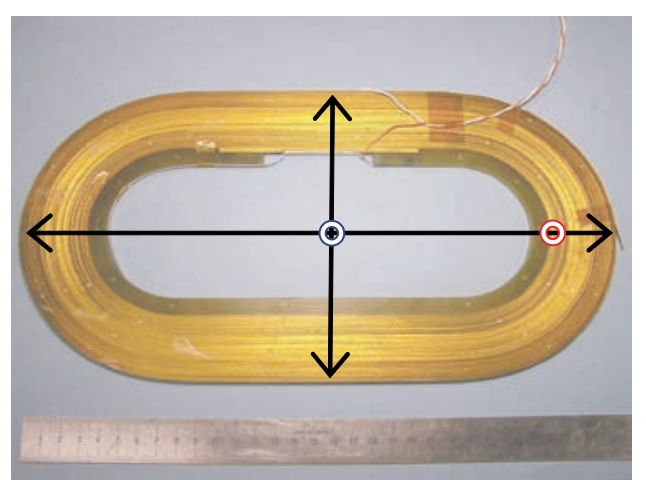

Fig. 12 Racetrack shape RE coil

$\left(\begin{array}{l}\text { Magnetic field sensor : } \\ \text { () Coil center } \\ \text { (a) Most internal layer at center of curve part }\end{array}\right)$

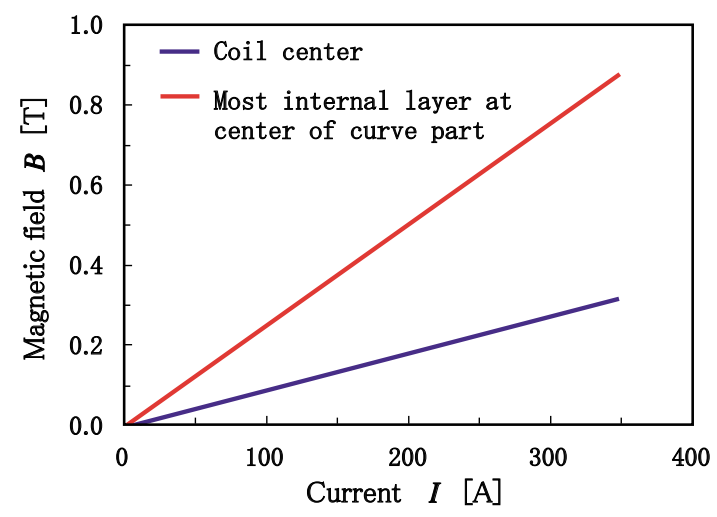

Fig. 13 Current dependence of magnetic field for RE coil (30 K)

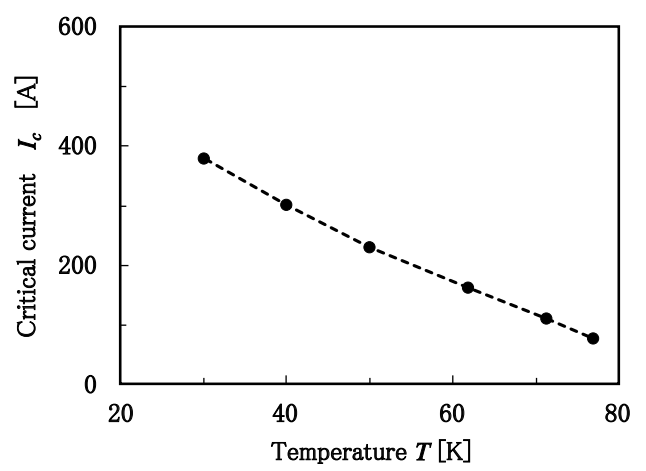

Fig. 14 Temperature dependence of critical current for RE coil

\section{Comparison of the transport current properties of RE wire and the RE coil}

When the magnetic field angle $\theta$ is constant, the magnetic field $B$ and the temperature $T$ determine the $I_{c}$ of the coil. If $T$ is also given in addition to $\theta$, the $I_{c}$ value depends only on $B$. Here, $B$ is the magnetic field that the coil itself generates with transport current. Because of the dimensions of the coil's cross section, the influence of $B$ on the transport current characteristics of the wire that constitutes the coil reaches its maximum at the two points shown in Fig. 15. Briefly, the $I_{c}$ of the coil can be calcu- 


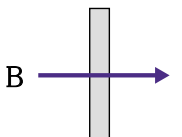

Point of $B_{\max }(\theta=90 \mathrm{deg})$

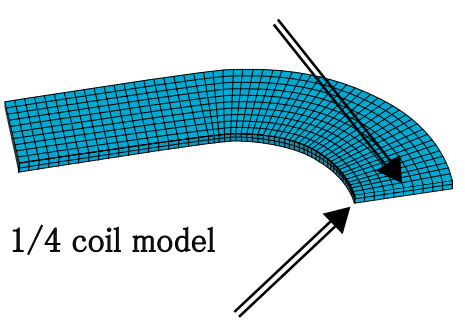

Point of $\operatorname{Bmax}(\theta=0 \mathrm{deg})$

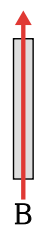

Fig. 15 Two positions where effect of magnetic field on transport current performanse is remarkable

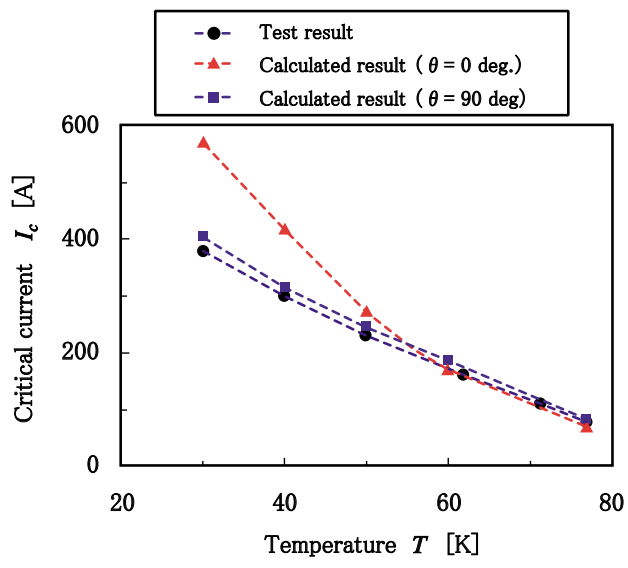

Fig. 16 Temperature dependence of critical current for RE coil

(Comparison of test result and calculated result)

lated by $B$ at these two points.

Using the results of the test on the wire's transport current characteristics and the calculation method above, we calculated $I_{c}$ at the points of maximum magnetic field $B_{\max }$, where $\theta=90$ degrees and $\theta=0$ degrees. Figure 16 shows a comparison of the test results and the calculated results for the temperature dependence of $I_{c}$ in the RE coil. Consistency was found between the test results and the calculated results for $\theta=90$ degrees, as shown in Fig. 16. This outcome indicates that if the transport current characteristics of the wire and the dimensions of the coil are known beforehand, it is possible to predict the transport current characteristics of the coil. This estimation method is also applicable to the study of coil design with a specific level of performance.

For studies on the applicability of RE wire to superconducting magnets for maglev system, both evaluation testing for the latest $\mathrm{RE}$ wire and analysis using the method to estimate coil transport current characteristics from those of the wire are important.

\section{Summary}

Several advantages, such as a simpler structure and a higher reliability, are gained from the application of $\mathrm{RE}$ wire to on-board superconducting magnets for maglev system. The points of this study can be summarized as follows:

(1) Using a system to evaluate the transport current characteristics of $\mathrm{RE}$ wire, its critical current characteristics in relation to temperature, magnetic field, magnetic field angle and bend diameter were verified.

(2) The temperature dependence of the critical current characteristics in a small racetrack-shaped $\mathrm{RE}$ coil made of RE wire was experimentally ascertained to complete the confirmation.

(3) A method of estimating the transport current characteristics of the RE coil using the characteristics of the constituent wire and the coil dimensions was described.

The authors plan to continue evaluation testing for the latest $\mathrm{RE}$ wire and utilize the results to enable the application of RE wire in maglev system.

This work was financially supported by the Japanese Government's Ministry of Land, Infrastructure, Transport and Tourism.

\section{References}

[1] Nagashima, K., Ogata, M., Miyazaki, Y., Hasegawa, H., Sasakawa, T., "Study of HTS magnet for maglev using RE-Ba-Cu-O wire (3)," abstracts of Cryogenic Society of Japan Conference, Vol.79, p.103, 2008 (in Japanese).

[2] Ogata, M., Nagashima, K., Miyazaki, Y., Iwamatsu, M., Yanase, Y., Yamashita, T., "A properties measuring system for HTS wires," RTRI Report, Vol.22, pp.2328, 2008 (in Japanese).

[3] Shiohara, Y., "Characteristics of 2nd generation high temperature superconducting wire and R\&D trends," IEEJ Journal, Vol.126, pp.268-271, 2006 (in Japanese). 